\title{
Designing Social Support Enabling Mobile Application For New Mothers
}

\author{
Annu Prabhakar \\ University of Cincinnati/ \\ Indiana University \\ Bloomington, Indiana \\ asprabha@indiana.edu \\ Xiao Liang \\ Indiana University \\ Bloomington, Indiana \\ liang25@umail.iu.edu
}

\author{
Anup Bharadwaj \\ Indiana University \\ Bloomington, Indiana \\ anupbhar@indiana.edu
}

\author{
Katie Siek \\ Indiana University \\ Bloomington, Indiana \\ ksiek@indiana.edu
}

\author{
Sujith Shivaprakash \\ Indiana University \\ Bloomington, Indiana \\ sujishiv@indiana.edu
}

\author{
Kay Connelly \\ Indiana University \\ Bloomington, Indiana \\ connelly@indiana.edu
}

\begin{abstract}
Social support plays an important role in the well-being of new mothers and their infants. Lack of social support is a risk factor for postpartum depression (PPD) in new mothers. The long term goal of our research is to design and develop a socio-technical system to facilitate social support for new mothers. To this end, we identified common needs of new mothers using semi-structured interviews and discussions in closed Facebook groups consisting of new mothers. Informed by the identified needs, we designed and developed the prototype of a mobile application called Mom's Circle, in which a new mom can enroll a community of helpers who agree to help her with common instrumental needs. In this paper, we discuss key findings from the needs assessment study, an overview of Mom's Circle App and results from the initial field deployment of Mom's Circle.
\end{abstract}

\section{CCS CONCEPTS}

-Information systems $\rightarrow$ Mobile information processing systems; • Human-centered computing $\rightarrow$ Mobile phones;

\section{KEYWORDS}

Mothers, Social Support, PPD, Android App, Mobile App, Digital Motherhood, Motherhood, Maternal Health, Mom's Circle

\section{INTRODUCTION}

Becoming a new mother is a joyous and rewarding experience for most women. However, 10 to $20 \%$ of new mothers are diagnosed with postpartum depression (PPD) [10]. Postpartum depression can have adverse long-term effects on mothers and their infants. For mothers, PPD can lead to chronic recurrent depression. For infants, mothers' ongoing depression can contribute to emotional, behavioral, cognitive and interpersonal problems later in life.

There are multiple risk factors associated with PPD. They include history of major depression, psycho-social stress, and inadequate social support [3]. Common types of treatment for PPD such as psychotherapy, antidepressant treatments and estrogen treatment [13] do not address the lack of social support which is a major risk factor for PPD.

The connection between lack of social support and PPD has been established for decades [6]. However, there has not been significant attempt in designing socio-technical interventions to facilitate social support for new mothers. Our work contributes towards addressing this issue by designing a novel socio-technical solution to facilitate social support between new mothers and their potential supporters. Our main contributions are:

1. Confirmation of instrumental help (explained in Related work section) as an important support type for new mothers.

2. Description of a working prototype of a support-enabling mobile application as a starting point for further research and development.

3. Discussion of the initial evaluation of the prototype.

\section{RELATED WORK}

Our work builds upon previous research across three main areas: social support and health outcome, role of social support in maternal wellness, and existing socio-technical solutions for mothers.

\subsection{Social support and health outcomes}

The relationship between social support and better health outcome has been studied over the decades. There are three main categories of support; emotional support, informational support and instrumental support [7]. Emotional support is acting as a confidant for someone, informational support is providing suggestions or useful information, and instrumental support is offering help or assistance in a tangible way, such as helping to do laundry, cook dinner, or clean. Women consider instrumental support essential for their physical and emotional well-being after child birth [12, 14]. A study by Cohen found that social support can improve an individual's 
ability to cope with stressful life events such as transition to motherhood [5].

\subsection{Role of social support in maternal wellness}

Researchers have identified social support as one resource that has shown to be effective in helping women cope with a range of stressors following childbirth [2, 17]. In 1981, Crokenberg conducted a longitudinal study of temperamentally difficult infants and their families. She found that mothers of irritable babies who had high levels of social support were able to establish more secure attachments with their infants than women with low levels of social support [8].

The link between the lack of social support and PPD crosses cultures. A study in the United States provided evidence for a relation between low levels of social support and PPD [4]. A study in 2005, of Hong Kong Chinese women also found a significant inverse relationship between PPD and social support [11]. Another study showed that adequate social support leads to decreased depression in both African American and European American women [15] Women consider instrumental support (such as help with cooking, laundry and cleaning) essential for their physical and emotional well-being after childbirth [12].

\subsection{Socio-technical solutions for mothers}

There are technology solutions that help women care for their infants. Hayes et. al designed and developed Estrellita, a tool to support parents of preterm infants to track health data [9]. Suh et. al developed a system called @BabySteps for allowing parents who use Twitter to track and respond to tweets about developmental milestones using a special hashtag syntax [16]. Balaam et. al developed FeedFinder, a location-mapping mobile application that helps breastfeeding women in finding, reviewing and sharing public breastfeeding places with other breastfeeding women [1].

\section{METHODS}

We conducted needs analysis to investigate the support needs of new mothers. Informed by it's findings we designed the prototype application.

\subsection{Needs Analysis}

Needs analysis consisted of literature review, a closed Facebook group study, and interviews of new moms. For the purpose of our study, we define new mother as a mother who has a child less than 4 months old. The goals of the literature review was to understand the existing research on connection between social support and health outcomes, mothers' social support needs, and existing socio-technical systems for social support for mothers. Using closed Facebook groups, we engaged 48 women in various activities to understand their support structure and support needs. We also conducted interviews with 12 women to further investigate their support needs.

The results of needs analysis are summarized below:

1. New mothers depend on their partners, mother figures, and close friends the most for help as they transition to motherhood. 2. New mothers value instrumental support the most during the initial months of motherhood.

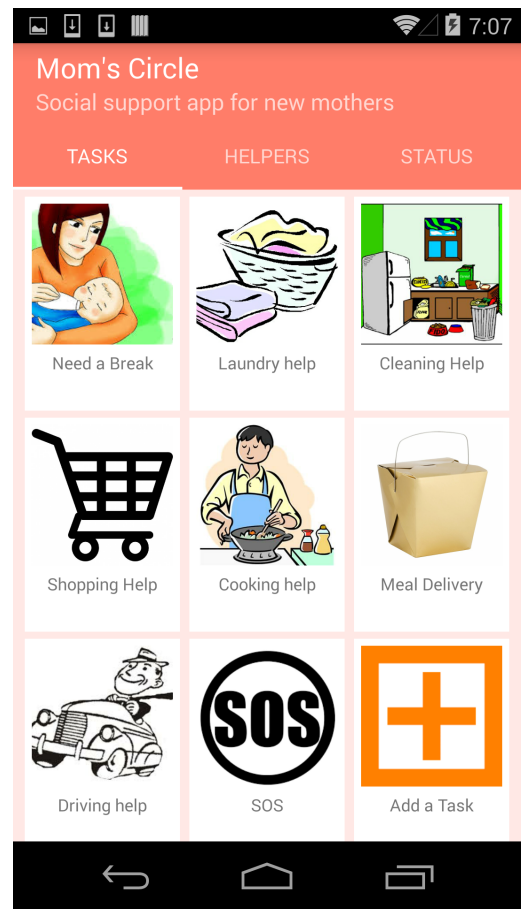

Figure 1: Mom's Circle main screen.

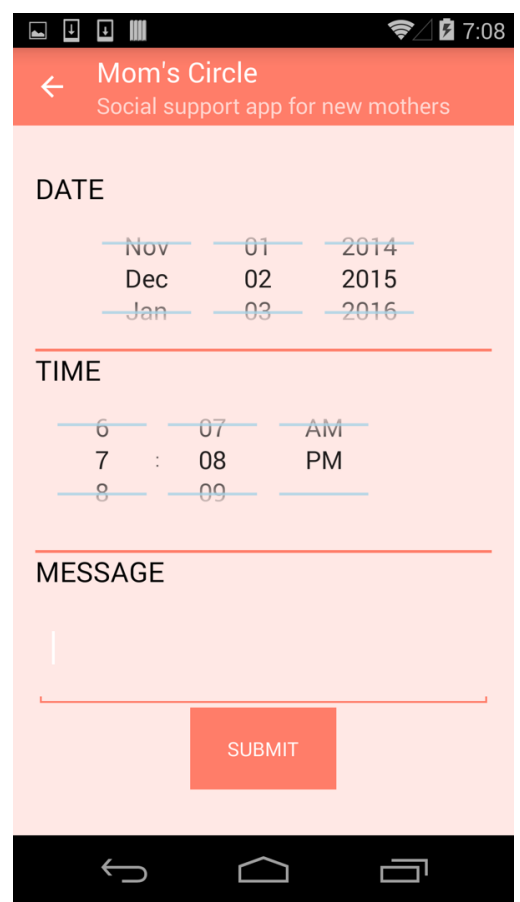

Figure 2: Date/Time picker screen. 


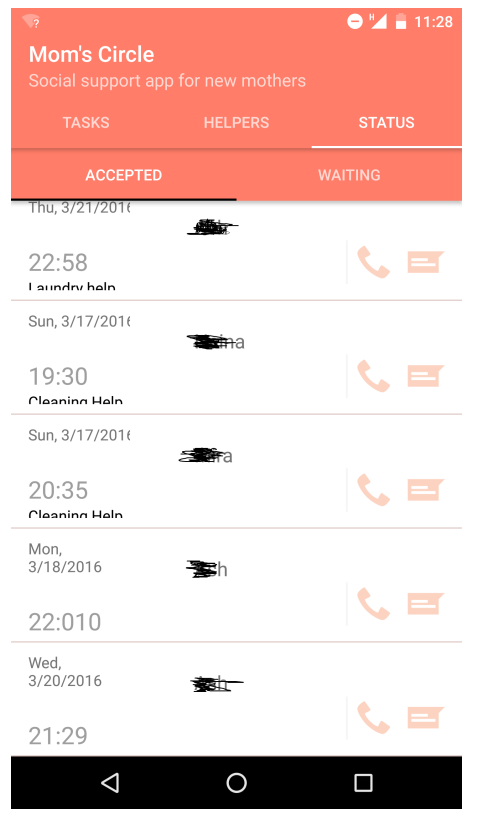

Figure 3: Tasks status screen.

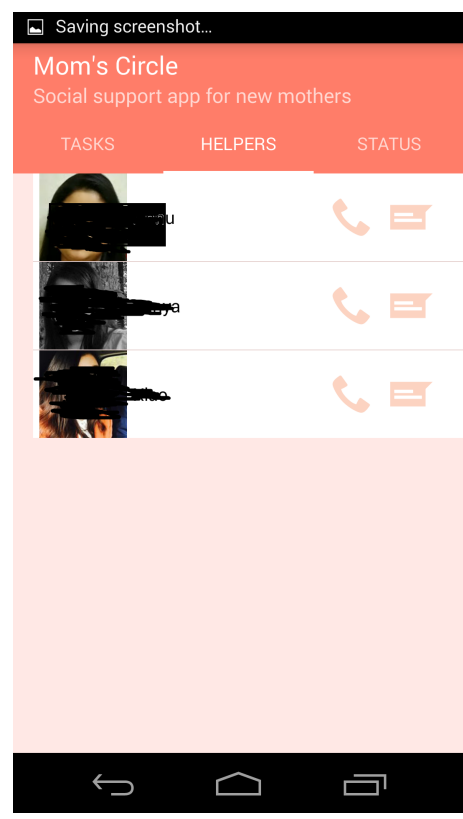

Figure 4: List of Mom's helpers.

3. There is a lack of socio-technical systems that facilitate supportiveinteractions between new mothers and their potential supporters.

Informed by these findings we designed and developed a working prototype of a mobile App, Mom's Circle, to facilitate interactions between new mothers and their supporters.

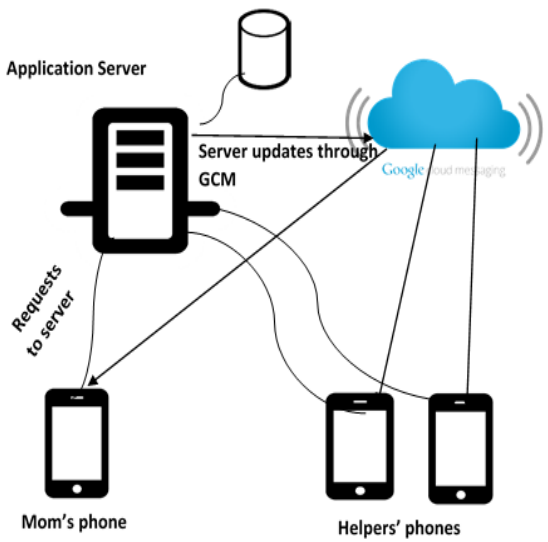

Figure 5: Architecture of Mom's Circle App.

\section{THE PROTOTYPE: MOM'S CIRCLE}

Mom's circle is an Android application which helps new mothers to ask for instrumental support from her supporters. When a mother opens Mom's Circle App, she will see the main screen (Figure 1) where she can choose one of 9 clickable tiles that initiates a help request. When she selects a tile, the date/time picker screen (Figure 2) appears, from where she can select the date and time of the needed help. The request will go to all of her helpers (who are people already in a mom's social circle and have installed the App in their phones) and generates a sound alert on their phones. They can choose to accept or ignore the request. If a helper accepts the task, it will disappear from all other helpers' screens. The mom's view will show the request as accepted with the information of the helper who accepted the request (Figure 3). The tasks that are waiting to be accepted will appear under "waiting" tab. For an emergency or for requesting a task that does not have a specific interface tile, there is an 'SOS' tile that enables the mom to text or call a helper. When she clicks on "SOS" it takes her directly to the helpers tab. From there she can initiate a phone call or text (Figure 4).

\subsection{App architecture design}

Mom's Circle is implemented as an Android app for mothers and their helpers. There is a version for moms and a version for helpers. On the back-end, there is an application server with PHP scripts and a MYSQL database for processing and storing data. The app communicates with the server to get information from the database. Any tasks created by the mom in her app will be added to the database on the server, and these new updates are pushed to her helpers' phones in the form of a mobile notification. We used the Google Cloud Messaging (GCM) API to deliver the server updates to their respective mobile devices. Figure 5 shows the architecture design of Mom's Circle.

\section{EVALUATION}

We evaluated screen flow of the prototype and functionality (such as sending a request, receiving acknowledgement, texting a helper) through a task-based beta assessment. Three mothers and their 
three supporters used the App for a week to evaluate it. We got positive feedback from all of them. Mothers liked the idea of giving choice to helpers. One participant said: 'I love this because you are able to specify what you need help with without actually having to ask for help. For a non-confrontational person like myself, that is the only way I could ask for help.'

We also received many constructive feedback on features which we plan to incorporate into the next stages of our research. For example a participant conveyed the following suggestions: 'It would be nice if this app could pull info from the contacts and events already on your phone, as well as integrate with social media, if you wanted. I would also like if the helpers could create their own entries and submit those to you for approval. This would help with scheduling and could be used to plan for visitors.'

\section{NEXT STEPS}

Our next step is to evaluate the system in situ, with more participants for an extended period of time. We plan to use experience sampling and interviews to collect moms' feedback and suggestions for improvements to Mom's Circle. In order to investigate the App's effect on support interactions and wellness, we will analyze the interaction between moms and their supporters, qualitatively and quantitatively, while they use Mom's Circle.

\section{CONCLUSION}

Social support can prevent the onset of postpartum depression in new mothers. Our research revealed that currently there are no socio-technical systems available to facilitate instrumental social support for new mothers. We developed an application prototype, Mom's Circle, that helps new mothers ask and receive social support. With this study, we encourage Pervasive health community to actively engage in designing and developing novel socio-technical systems to help improve the well-being of new mothers. We also invite the community to provide feedback on Mom's Circle and provide guidance for further development of the application.

\section{REFERENCES}

[1] Madeline Balaam, Rob Comber, Ed Jenkins, Selina Sutton, and Andrew Garbett. 2015. Feedfinder: a location-mapping mobile application for breastfeeding women. In Proceedings of the 33rd Annual ACM Conference on Human Factors in Computing Systems (CHI '15). ACM, Seoul, Republic of Korea, 1709-1718. ISBN: 978-1-4503-3145-6. DOI: 10.1145/2702123.2702328. http://doi.acm.org/10. 1145/2702123.2702328.

[2] Barbara Ballinger, Dorothy E Buckley, Graham J Naylor, and David A Stansfield 1979. Emotional disturbance following childbirth: clinical findings and urinary excretion of cyclic amp (adenosine 3'5'cyclic monophosphate). Psychological Medicine, 9, 02, 293-300.

[3] Cheryl Tatano Beck. 2001. Predictors of postpartum depression: an update. Nursing research, 50, 5, 275-285.

[4] Odette Bernazzani et al. 2004. Contextual assessment of the maternity experience: development of an instrument for cross-cultural research. The British Journal of Psychiatry, 184, 46, s24-s30.

[5] Sheldon Cohen. 2004. Social relationships and health. American Psychologist, 59, 8, 676-684. ISSN: 1520-6629.

[6] Nancy L Collins, Christine Dunkel-Schetter, Marci Lobel, and Susan C Scrimshaw. 1993. Social support in pregnancy: psychosocial correlates of birth outcomes and postpartum depression. Journal of personality and social psychology, 65, 6, 1243.

[7] Nancy L Collins, Christine Dunkel-Schetter, Marci Lobel, and Susan CM Scrimshaw. 2004. Social support in pregnancy: psychosocial correlates of birth outcomes and postpartum depression. Close relationships: Key readings, 35.
[8] Susan B Crockenberg. 1981. Infant irritability, mother responsiveness, and social support influences on the security of infant-mother attachment. Child development, 857-865.

[9] Gillian R. Hayes, Karen G. Cheng, Sen H. Hirano, Karen P. Tang, Marni S. Nagel, and Dianne E. Baker. 2014. Estrellita: a mobile capture and access tool for the support of preterm infants and their caregivers. ACM Trans. Comput.Hum. Interact., 21, 3, Article 19, (June 2014), 19:1-19:28. ISSN: 1073-0516. DoI: 10.1145/2617574. http://doi.acm.org/10.1145/2617574.

[10] Ann Josefsson, Göran Berg, Conny Nordin, and Gunilla Sydsjö. 2001. Prevalence of depressive symptoms in late pregnancy and postpartum. Acta obstetricia et gynecologica Scandinavica, 80, 3, 251-255.

[11] Sharron SK Leung, Ida M Martinson, and David Arthur. 2005. Postpartum depression and related psychosocial variables in hong kong chinese women: findings from a prospective study. Research in nursing \& health, 28, 1, 27-38.

[12] Rennie Negron, Anika Martin, Meital Almog, Amy Balbierz, and Elizabeth A Howell. 2013. Social support during the postpartum period: mothersfffdfffdfffd views on needs, expectations, and mobilization of support. Maternal and child health journal, 17, 4, 616-623.

[13] T Pearlstein, M Howard, A Salisbury, and C Zlotnick. 2009. Postpartum depression. American journal of obstetrics and gynecology, 200, 04, 357-364.

[14] Annu Sible Prabhakar et al. 2017. "let me know if you need anything": support realities of new mothers. In Proceedings of the 11th EAI International Conference on Pervasive Computing Technologies for Healthcare (PervasiveHealth '17). ACM, Barcelona, Spain, 31-40. ISBN: 978-1-4503-6363-1. DOI: 10.1145/3154862.3154863. http://doi.acm.org/10.1145/3154862.3154863.

[15] Christian Ritter, Stevan E Hobfoll, Justin Lavin, Rebecca P Cameron, and Michael R Hulsizer. 2000. Stress, psychosocial resources, and depressive symptomatology during pregnancy in low-income, inner-city women. Health Psychology, 19, 6, 576.

[16] Hyewon Suh, John R. Porter, Alexis Hiniker, and Julie A. Kientz. 2014. @babysteps: design and evaluation of a system for using twitter for tracking children's developmental milestones. In Proceedings of the SIGCHI Conference on Human Factors in Computing Systems (CHI '14). ACM, Toronto, Ontario, Canada, 2279-2288. ISBN: 978-1-4503-2473-1. DOI: 10.1145/2556288 . 2557386. http: //doi.acm.org/10.1145/2556288.2557386.

[17] Lois Wandersman, Abraham Wandersman, and Steven Kahn. 1980. Social support in the transition to parenthood. Journal of Community Psychology, 8, 4, 332-342. ISSN: 1520-6629. 